\title{
Episodic Ataxia Mutations in Kv1.1 Alter Potassium Channel Function by Dominant Negative Effects or Haploinsufficiency
}

\author{
Patricia Zerr, ${ }^{1}$ John P. Adelman, ${ }^{1}$ and James Maylie ${ }^{2}$ \\ 1 Vollum Institute and 'Department of Obstetrics and Gynecology, Oregon Health Sciences University, Portland, Oregon \\ 97201
}

Subunits of the voltage-gated potassium channel Kv1.1 containing mutations responsible for episodic ataxia (EA), a human inherited neurological disease, were expressed in Xenopus oocytes. Five EA subunits formed functional homomeric channels with lower current amplitudes and altered gating properties compared with wild type. Two EA mutations located in the first cytoplasmic loop, R239S and F249I, yielded minimal or no detectable current, and Western blot analysis showed reduced protein levels. Coinjection of equal amounts of EA and wildtype mRNAs, mimicking the heterozygous condition, resulted in current amplitudes and gating properties that were intermediate between wild-type and EA homomeric channels, suggesting that heteromeric channels are formed with a mixed stoichiometry of EA and wild-type subunits. To examine the relative contribution of EA subunits in forming heteromeric EA and wild-type channels, each EA subunit was made insensitive to TEA, TEA-tagged, and coexpressed with wild-type subunits. TEA-tagged R239S and F249l induced the smallest shift in TEA sensitivity compared with homomeric wild-type channels, whereas the other TEA-tagged EA subunits yielded TEA sensitivities similar to coexpression of wild-type and TEA-tagged wild-type subunits. Taken together, these results show that the different mutations in Kv1.1 affect channel function and indicate that both dominant negative effects and haplotype insufficiency may result in the symptoms of EA.

Key words: episodic ataxia; neurological disease; K channel; oocyte expression; haploinsufficiency; dominant negative effects
The genetic lesions responsible for many inherited neuromuscular and ataxic syndromes have been identified and in many cases occur in the coding sequence of an ion channel gene (Kraus and McNamara, 1995; Ackerman and Clapham, 1997; Greenberg, 1997). In some instances, the biophysical properties of the mutant channel have been examined, but for many the underlying molecular mechanism has not been established.

Episodic ataxia (EA) is an autosomal dominant neurological disorder, affecting both central and peripheral nerve functions, with symptomatic attacks of imbalance and uncontrolled movements (Ashizawa et al., 1983). The attacks of ataxia may be induced by physical or emotional stress; they usually last several minutes and may occur several times a day. Although the symptoms are varied between and within families, presumably reflecting the outbred nature of the human population, two symptoms are always observed: an ataxic gait during attacks and myokymia, characterized by a continuous muscle activity, which is presented as a rhythmic electromyography activity with a pattern of repeated duplets and multiplets (Gancher and Nutt, 1986; Brunt and Weeden, 1990).

Genetic linkage studies have localized the EA syndrome locus to chromosome 12p13 (Litt et al., 1994) and, subsequently KCNA1, the gene encoding the voltage-gated delayed rectifier $\mathrm{K}^{+}$channel Kv1.1, was identified as underlying EA (Browne et

\footnotetext{
Received Nov. 24, 1997; revised Jan. 20, 1998; accepted Jan 23, 1998.

This work was supported by National Institutes of Health grants to J.P.A. and J.M. and a Medical Research Foundation grant to J.M. P.Z. was supported in part by the Ministère des Affaires Etrangères de France and the Philippe Foundation. We thank Zhaoping Liu for helpful discussions and Chris T. Bond for the EA constructs.

Correspondence should be addressed to Dr. James Maylie, Department of Obstetrics and Gynecology, Oregon Health Sciences University, L-458, 3181 SW Sam Jackson Park Road, Portland, OR 97201.

Copyright (C) 1998 Society for Neuroscience $\quad 0270-6474 / 98 / 182842-07 \$ 05.00 / 0$
}

al., 1994). All of the mutations occur in positions highly conserved among the voltage-dependent $\mathrm{K}^{+}$channel superfamily. In each affected family, a different missense point mutation has been identified in the coding sequence of Kv1.1, and all affected individuals are heterozygous (Browne et al., 1994, 1995). $\mathrm{K}^{+}$channels are formed by the assembly of four subunits (MacKinnon, 1991), and if wild-type and EA alleles are expressed, both homomeric and heteromeric channels may be formed.

Our previous study showed that some of the EA alleles encode functional channels and indicated that the autosomal dominant phenotype may be attributed to heteromeric channel assembly of wild-type and EA subunits (Adelman et al., 1995). In this report we show that three alleles previously thought to encode nonfunctional subunits form functional channels when higher levels of expression are achieved. Five EA subunits that form functional but aberrant channels coassembled with wild-type subunits and showed different dominant negative potencies when expressed at a 1:1 ratio. Two EA subunits with the least dominant negative impact on heteromeric channels also have markedly reduced protein levels. These results show that different mechanisms may underlie altered $\mathrm{K}^{+}$channel function in EA-affected individuals.

\section{MATERIALS AND METHODS}

Xenopus care and handling were in accordance with the highest standards of institutional guidelines. Frogs underwent no more than two surgeries, separated by at least 3 weeks. To isolate oocytes, frogs were anesthetized with an aerated solution of 3-aminobenzoic acid ethyl ester. Standard recording solution contained (in $\mathrm{mM}$ ): $96 \mathrm{NaCl}, 2 \mathrm{KCl}, 1 \mathrm{MgCl}_{2}, 1.8$ $\mathrm{CaCl}_{2}$, and $5 \mathrm{HEPES}, \mathrm{pH}$ 7.4. All chemicals were from Sigma (St. Louis, $\mathrm{MO}$ ). Two-electrode voltage-clamp recordings were performed at room temperature with a Geneclamp 500 amplifier (Axon Instruments, Burlingame, CA) interfaced to a Macintosh Quadra 800 computer. Linear leak and capacitance currents were corrected with a P/4 leak subtraction procedure. Data collection and analysis were performed using Pulse, 
A

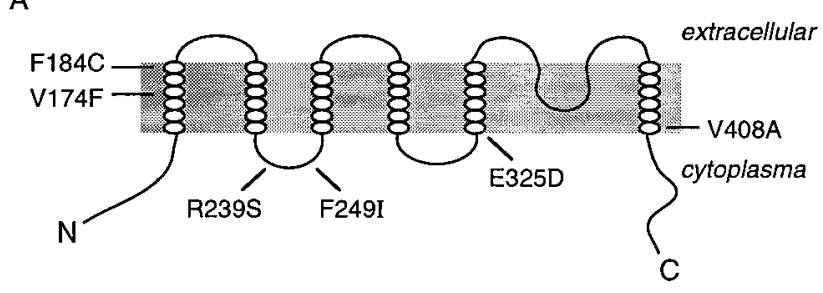

B

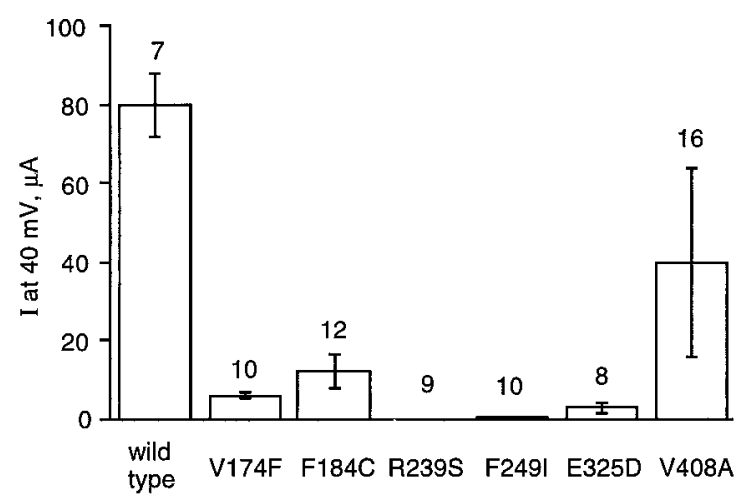

Figure 1. A, Schematic representation of the membrane topology of Kv1.1 subunits. The positions of the six EA point mutations studied are indicated. $B$, Histogram showing current amplitudes recorded from oocytes injected with wild-type and EA mRNAs equilibrated as described in Materials and Methods. Currents were measured at the end of a $200 \mathrm{msec}$ pulse to $40 \mathrm{mV}$. Error bars indicate SD; the number of cells recorded is indicated in each case.

PulseFit (Heka), IGOR (Wavemetrics), and KaleidaGraph (Synergy Software). Statistical significance was determined by an unpaired Student's $t$ test, and $p<0.01$ was considered significant.

Human Kv1.1 cDNA was cloned into the vector $\mathrm{pS}^{-}$(Promega, Madison, WI). Site-directed mutagenesis, nucleotide sequencing, and in vitro mRNA synthesis were performed as described previously (Adelman et al., 1995). Equal amounts of DNA were linearized and transcribed using common pools of reagents; mRNAs were initially evaluated by denaturing gel electrophoresis and ethidium bromide stain as well as by spectrophotometer. For rigorous quantification, mRNAs were diluted in triplicate (1:20 dilution) and dot-blotted in duplicate onto a GeneScreen filter (DuPont NEN, Boston, MA), which was then hybridized to a Kv1.1specific radiolabeled oligonucleotide. To assure saturating hybridization, the radiolabeled oligonucleotide was present in vast excess to the target mRNA (>200-fold), and hybridization was allowed to proceed overnight in a small volume. After washes, hybridization signals were quantified with a scintillation counter or imaged with a PhosphorImager (Molecular Dynamics, Sunnyvale, CA), and the density was determined with NIH-
Image 1.59 software. Based on this quantification, mRNA amounts were adjusted to yield the ratios indicated in the text. Oligonucleotides were purchased from Genosys.

Western blots were performed as described (Tucker et al., 1996). Only paired groups of oocytes with wild-type or EA mRNAs in which wildtype currents were $>30 \mu \mathrm{A}$ were used. Total oocyte membranes were prepared using a standard method (Geering et al., 1989). Briefly, 25 oocytes were washed twice, suspended in $1 \mathrm{ml}$ of PBS containing $0.1 \mathrm{~mm}$ phenylmethylsulfonyl fluoride and $5 \mu \mathrm{g} / \mathrm{ml}$ leupeptin, aprotinin, and pepstatin $\mathrm{A}$, and homogenized, first by five passages through a 28 gauge needle and then two passages through a 27 gauge needle. The homogenates were centrifuged repeatedly at $2000 \times g$ for $10 \mathrm{~min}$ at $4^{\circ} \mathrm{C}$ until all yolk granules and melanosomes were pelleted, typically three or four times. The final supernatant was pelleted at $60,000 \times g$ for $30 \mathrm{~min}$ at $4^{\circ} \mathrm{C}$ to generate a total membrane fraction devoid of yolk granules. This membrane pellet was resuspended in $25 \mu \mathrm{l}$ of $50 \mathrm{~mm}$ Tris, $150 \mathrm{~mm} \mathrm{NaCl}$,

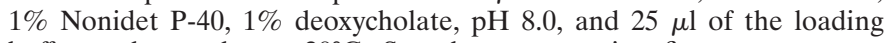
buffer and stored at $-20^{\circ} \mathrm{C}$. Samples representing five oocytes were subjected to SDS-PAGE using a 10\% resolving gel and 3\% stacking gel in a Bio-Rad (Hercules, CA) Miniprotean II apparatus. Proteins were transferred to nitrocellulose filters, and Western blot analysis was performed using an anti-mouse Kv1.1 polyclonal antibody (a generous gift from Dr. Bruce Tempel, Department of Otolaryngology, University of Washington, Seattle, WA). Antibodies were detected using the ECL detection system (Amersham, Arlington Heights, IL) according to the manufacturer's instructions, and signals were quantified using IP labgel software (Molecular Dynamics).

\section{RESULTS}

\section{Expression of EA subunits in Xenopus oocytes}

The positions of the six EA mutations introduced into the human Kv1.1 cDNA are shown in Figure $1 A$. To determine the functional consequences of the EA mutations, equal amounts of wild-type and EA mRNAs were separately injected into Xenopus oocytes, and currents were examined in the two-electrode voltage-clamp configuration. As described previously, oocytes expressing F184C and V408A subunits had reduced current amplitudes measured at $40 \mathrm{mV}$ compared with oocytes expressing wild-type subunits (Fig. 1B) (Adelman et al., 1995). V174F, R239S, F249I, and E325D had been reported previously as not forming functional channels. However, when higher levels of expression were achieved, either by injecting more mRNA or by waiting longer after injection, currents were detected with V174F, F249I, and E325D, although in all three cases the current amplitudes were 10 - to 100 -fold lower than that of wild type (Fig. 1B). In contrast, oocytes expressing R239S never yielded currents different from noninjected oocytes. The current amplitude at $40 \mathrm{mV}$ for each EA mutant relative to wild type is summarized in Table 1.

As described previously (Adelman et al., 1995), F184C currents showed a shift in the voltage dependence of $\sim 25 \mathrm{mV}$ with slowed

Table 1. Biophysical parameters of homomeric wild-type and the five conducting EA mutants

\begin{tabular}{|c|c|c|c|c|c|c|c|c|}
\hline & \multirow{2}{*}{$\begin{array}{l}\text { Relative } \\
\text { amplitudes }\end{array}$} & \multicolumn{2}{|c|}{ Voltage-dependent parameters } & \multicolumn{2}{|l|}{ Activation } & \multicolumn{2}{|l|}{ Deactivation } & \multirow{2}{*}{$\begin{array}{l}\text { C-type } \\
\text { inactivation } \\
I_{\text {final }} / I_{\text {peak }} \\
\end{array}$} \\
\hline & & $\mathrm{V}_{1 / 2}(\mathrm{mV})$ & $k(\mathrm{mV})$ & $\tau_{\mathrm{V}_{1 / 2}}(\mathrm{msec})$ & $k(\mathrm{mV})$ & $\tau_{\mathrm{V}_{1 / 2}}(\mathrm{msec})$ & $k(\mathrm{mV})$ & \\
\hline Wild type & 100.0 & $-28.8 \pm 2.3(6)$ & $8.1 \pm 0.9$ & $14.6 \pm 3.7(5)$ & $30.1 \pm 2.8$ & $22.8 \pm 2.3(5)$ & $26.2 \pm 2.5$ & $0.70 \pm 0.01(10)$ \\
\hline V174F & 7.6 & $6.3 \pm 0.7(8)^{*}$ & $6.1 \pm 0.6^{*}$ & $9.8 \pm 2.2(6)$ & $31.3 \pm 4.3$ & $26.0 \pm 3.8(3)$ & $28.5 \pm 2.4$ & $0.60 \pm 0.08(6)^{*}$ \\
\hline F184C & 15.1 & $-2.9 \pm 1.4(11)^{*}$ & $7.1 \pm 0.7$ & $28.2 \pm 0.7(5)^{*}$ & $28.9 \pm 0.7$ & $27.4 \pm 2.0(12)^{*}$ & $32.6 \pm 6.6$ & $0.74 \pm 0.02(10)^{*}$ \\
\hline F249I & 1 & $-29.4 \pm 3.4(6)$ & $6.2 \pm 0.9^{*}$ & $15.6 \pm 3.7(5)$ & $31.4 \pm 4.5$ & $44.7 \pm 3.9(4)^{*}$ & $23.2 \pm 0.7$ & $0.74 \pm 0.03(2)^{*}$ \\
\hline E325D & 3.8 & $31.6 \pm 0.8(6)^{*}$ & $17.5 \pm 0.7^{*}$ & $2.0 \pm 0.3(8)^{*}$ & $205 \pm 92 *$ & $3.5 \pm 1.0(5)^{*}$ & $54.2 \pm 10.3^{*}$ & $0.52 \pm 0.05(10)^{*}$ \\
\hline V408A & 49.8 & $-28.4 \pm 2.5(6)$ & $7.5 \pm 0.4$ & $7.8 \pm 1.2(8)^{*}$ & $39.6 \pm 5.2^{*}$ & $2.1 \pm 0.3(3)^{*}$ & $18.6 \pm 3.0^{*}$ & $0.15 \pm 0.01(4)^{*}$ \\
\hline
\end{tabular}

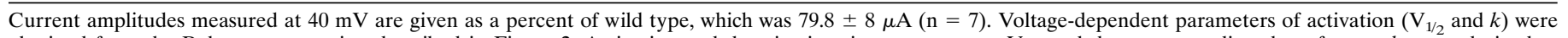

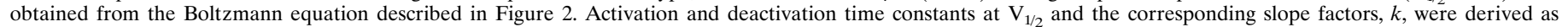
described in Figure 2. C-type inactivation was measured at $20 \mathrm{mV}$ and presented as the ratio $\mathrm{I}_{\text {final }} / \mathrm{I}_{\text {peak. }}$. Data are presented as mean $\pm \mathrm{SD}$ (number of cells).

*Significance of $p<0.01$ compared with wild type. 
A V174F
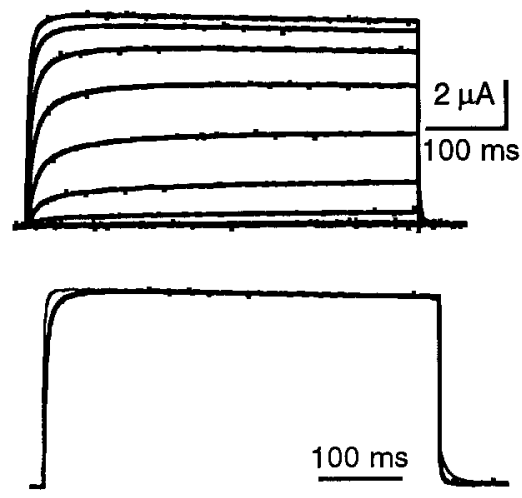

Normalized Itail
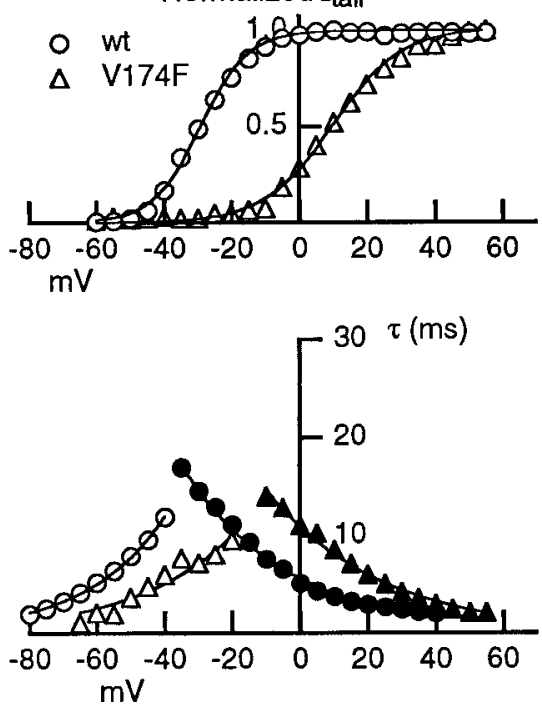

B
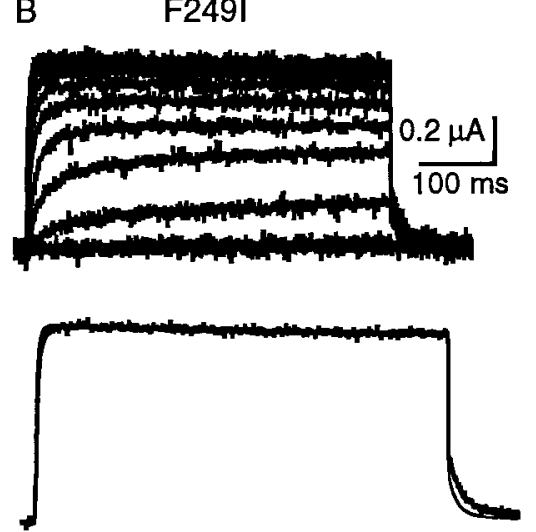

Normalized Itail
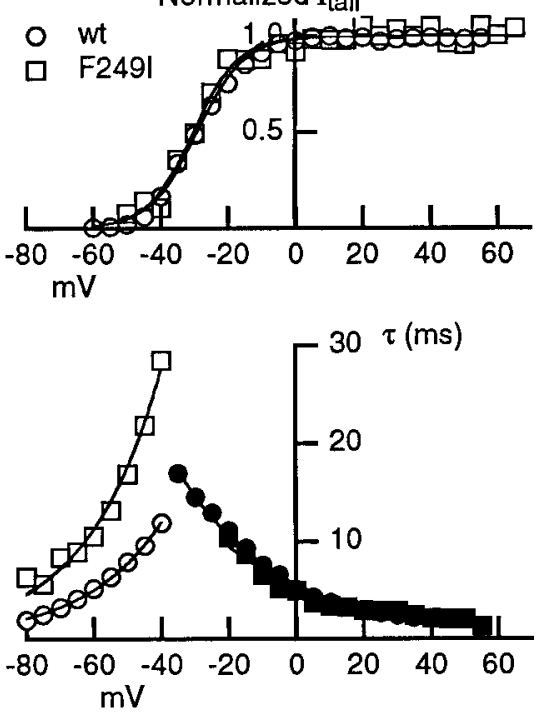

C
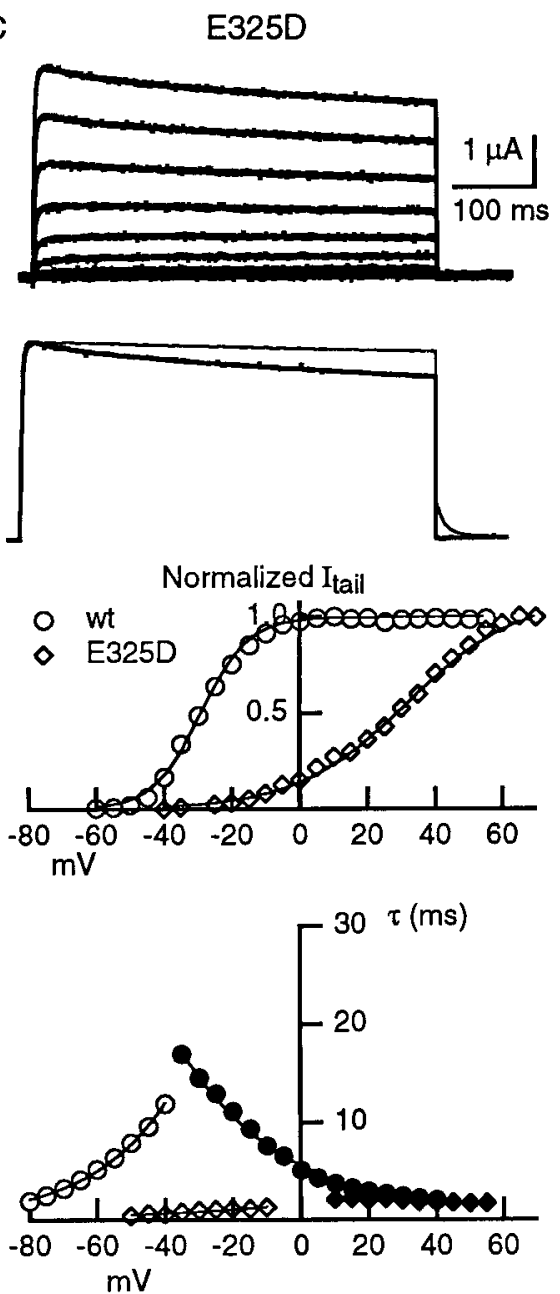

Figure 2. Characterization of V174F, F249I, and E325D. A, V174F; B, F249I; C, E325D. Top panels, Traces representing currents evoked from -60 to $50 \mathrm{mV}$ depolarizing pulses (increments of $10 \mathrm{mV}$ ) from a holding potential of $-80 \mathrm{mV}$. Tail currents were recorded at $-50 \mathrm{mV}$. Second panels, Current traces for each EA mutation (thick lines) were scaled and superimposed to a representative wild-type current trace (thin lines). Currents were evoked at $40 \mathrm{mV}$ from a holding potential of $-80 \mathrm{mV}$, and tail currents were recorded at $-50 \mathrm{mV}$. Third panels, Voltage dependence of activation from a single representative experiment. The normalized tail currents recorded at $-50 \mathrm{mV}$ (V174F, F249I) or at $-30 \mathrm{mV}$ (E325D) were plotted as a function of the preceding depolarizing potential $(-60$ to $65 \mathrm{mV})$. The tail currents were fitted with a single exponential, and the amplitude of the exponential was used to describe the tail currents. Data points were fitted according to the Boltzmann equation $I=1 /\left(1+\exp -\left(\mathrm{V}-\mathrm{V}_{1 / 2}\right) / k\right.$, where $\mathrm{V}_{1 / 2}$ is the potential of half-activation, and $k$ is a slope factor. Bottom panels, Time constants of activation ( filled symbols) and deactivation (open symbols) from a single representative experiment. Activation of currents was best described with a sum of two exponentials, and the time constant of the fast exponential was plotted here, whereas a single exponential was sufficient for the deactivation. Data points were fitted according to the equation $\tau=\tau_{\mathrm{V}_{1 / 2}} \cdot \mathrm{e}^{\left(\mathrm{V}-\mathrm{V}_{1 / 2}\right)} / k$, where $\tau_{\mathrm{V}_{1 / 2}}$ is the time constant at $\mathrm{V}_{1 / 2}$, and $k$ is the slope factor for the voltage dependence of the time constants.

activation kinetics, whereas V408A currents showed accelerated activation and deactivation kinetics and increased C-type inactivation (Table 1). To compare the effects of V174F, F249I, and E325D on gating, representative current traces recorded at 40 $\mathrm{mV}$ were overlaid and scaled to a representative wild-type current trace (Fig. 2). The comparison showed differences between these EA and wild-type channels that may reflect shifts in voltage dependence of activation and/or effects on channel kinetics. Therefore, the biophysical properties for these EA mutants were individually investigated.

\section{V174F shifts positively the voltage dependence of activation}

The voltage dependence of activation for V174F channels was examined by measuring tail currents at $-50 \mathrm{mV}$ after voltage commands to potentials between -60 and $65 \mathrm{mV}$. The normalized tail currents plotted against command potential showed that the voltage dependence of activation was shifted to positive potentials (Fig. 2A, third panel). The voltage dependence was quantified by fitting the data to a Boltzmann equation, which showed a shift of the voltage for half-maximal activation, $\mathrm{V}_{1 / 2}$, of $35 \mathrm{mV}$ compared with wild-type channels (Table 1). The overlaid traces in Figure $2 A$ show that $\mathrm{V} 174 \mathrm{~F}$ activation at $40 \mathrm{mV}$ was slower and deactivation at $-50 \mathrm{mV}$ was faster than in wild type. The kinetics of activation were measured by fitting the rising phase of the current traces evoked at different voltages with a double exponential; the fast time constant, which accounted for $>75 \%$ of the current, was plotted versus test potential (Fig. $2 \mathrm{~A}$, bottom panel). Deactivation rates were determined from tail currents evoked from -10 to $-70 \mathrm{mV}$ after a $100 \mathrm{msec}$ test pulse to $40 \mathrm{mV}$; deactivation was fitted with a single exponential, and the time constant was plotted versus tail potential (Fig. 2A, bottom panel). Throughout the voltage range, V174F activation was slower and deactivation was faster than in wild type. Because 


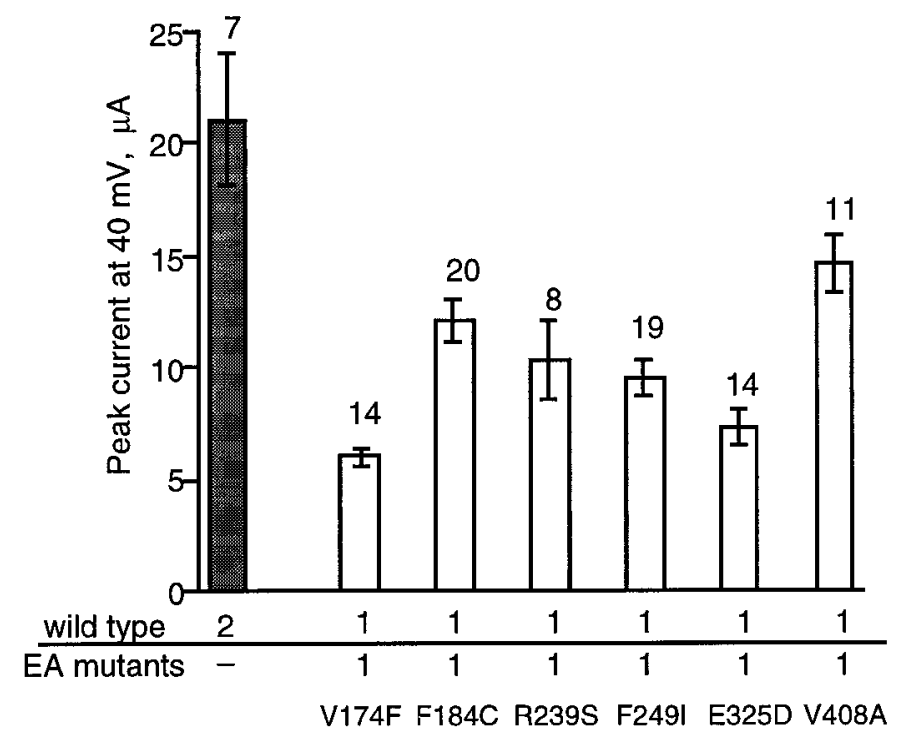

Figure 3. Coexpression of EA and wild-type subunits. Compared with oocytes injected with wild-type mRNA ( filled column), current amplitudes recorded from oocytes coinjected with wild-type and EA mRNAs (open columns) were reduced; relative amounts of mRNAs injected are indicated below each column. Currents were measured at the end of a 200 $\mathrm{msec}$ pulse to $40 \mathrm{mV}$. Error bars indicate SD; the number of cells recorded is indicated in each case.

the voltage dependence of current activation was affected, the kinetics of V174F were compared with those of wild type by determining the time constants of activation and deactivation at their respective $V_{1 / 2}$ values (Fig. 2). Interestingly, when compared at $\mathrm{V}_{1 / 2}$, the kinetics of activation and deactivation for $\mathrm{V} 174 \mathrm{~F}$ were similar to those for wild type (Table 1). These results showed that the effect of V174F on channel gating may be explained solely by a shift in voltage dependence. In addition, C-type inactivation, evaluated using a $10 \mathrm{sec}$ command pulse to $20 \mathrm{mV}$, was slightly larger than for wild type (Table 1).

\section{F249I slows the deactivation}

Analyses of F249I currents showed that the voltage dependence and kinetics of activation were similar to those of wild type (Fig. $2 B$, Table 1$)$. However, Figure $2 B$ shows that deactivation was slower and the corresponding time constant at $\mathrm{V}_{1 / 2}$ was increased approximately twofold without a significant change in voltage steepness (Table 1). C-type inactivation may be less pronounced for F249I (Table 1); however, the relatively small current amplitudes did not permit a clear analysis because of contamination with endogenous currents.

\section{E325D shifts positively the voltage dependence of activation and increases the rate of activation, deactivation, and C-type inactivation}

The voltage dependence of activation was examined by measuring tail currents at $-30 \mathrm{mV}$. Figure $2 C$ revealed a dramatic shift in the voltage dependence of E325D; the $\mathrm{V}_{1 / 2}$ was shifted $\sim 60 \mathrm{mV}$, and the steepness factor was increased approximately twofold, indicating a reduced voltage dependence (Table 1). As shown in Figure $2 C$ and summarized in Table 1 , time constants of activation and deactivation at $\mathrm{V}_{1 / 2}$ were faster compared with wild type by a factor of $\sim 6$. However, in contrast to the other EA mutants, the voltage dependence of the kinetics was considerably reduced compared with wild type. Similar to V408A, C-type inactivation of E325D was faster than for wild type, yielding an increased amount of inactivation (Table 1).

\section{Coexpression of wild-type and EA subunits}

EA is an autosomal dominant disease, and all known individuals are heterozygous. If both EA and wild-type Kv1.1 alleles are expressed to a similar extent in vivo, the EA and wild-type subunits may assemble according to a binomial distribution (MacKinnon, 1991; Kavanaugh et al., 1992), or they may only form homomeric EA and wild-type channels. Therefore, to examine the effects of expressing each EA subunit with wild-type subunit, equal amounts of EA and wild-type mRNAs were coinjected. Compared with currents recorded from oocytes injected with the same total amount of mRNA for wild type only (homozygous wild-type condition), the current amplitudes measured at $40 \mathrm{mV}$ were reduced in oocytes coinjected with half wild-type mRNA and half mRNA for each EA mutant (heterozygous condition) (Fig. 3). Comparison of current records obtained at a test pulse potential of $20 \mathrm{mV}$ and a tail potential of $-50 \mathrm{mV}$ showed that when some of the EA mutations were coexpressed with wild-type subunits, the currents demonstrated kinetics that were intermediate between those of homomeric EA and homomeric wild-type channels (Fig. 4). To examine these differences in more detail, the voltage dependence of activation, kinetics of activation at $20 \mathrm{mV}$ and deactivation at $-50 \mathrm{mV}$, and C-type inactivation at $20 \mathrm{mV}$ were assessed. Coinjection of R239S mRNA with wild-type mRNA yielded currents with voltage dependence and kinetics indistinguishable from those of homomeric wild type (Table 2). When coexpressed with wild type, V174F and F184C yielded activation kinetics intermediate between homomeric wild-type and the corresponding homomeric EA currents. Similarly, currents from oocytes coinjected with wild-type and E325D or V408A mRNAs yielded intermediate deactivation and C-type inactivation kinetics, and coexpression of wild-type and V174F, F184C, or E325D subunits showed an intermediate shift in activation voltage (Table 2 ). These results suggest that V174F, F184C, E325D, or V408A subunits coassemble with wild-type subunits, forming heteromeric channels with altered gating properties.

\section{Heteromeric channels containing $E A$ and wild-type subunits}

To confirm this hypothesis, EA subunits were altered in their sensitivity to external TEA (TEA-tagged EA subunits). The Shaker $\mathrm{K}^{+}$channel contains a tyrosine residue within the $\mathrm{P}$ loop that endows homomeric channels with high sensitivity to TEA $\left(K_{i}=0.2-0.4 \mathrm{~mm}\right)$; substitution of a valine at this position reduces sensitivity to TEA $>100$-fold (MacKinnon and Yellen, 1990). Substitution of a valine at the equivalent position in Kv1.1 (Y379V) similarly reduced the sensitivity to TEA $~ 100$ fold (Fig. 5, filled squares). In oocytes coexpressing TEAinsensitive (TEA-tagged) and TEA-sensitive subunits, the dose response to TEA reflects the number of tyrosinecontaining subunits within the channel (Kavanaugh et al., 1992). TEA-tagged EA or wild-type mRNA was coinjected with wild-type mRNA at a 1:1 ratio. The dose response to TEA was determined and compared with that from oocytes injected with wild type alone (Fig. 5). The data points for wild type (Fig. 5, open circles) were fitted with a single binding isotherm $\left(K_{i}=0.4 \mathrm{~mm}\right)$, and the data points for wild type and TEA tagged wild type were fitted with a binomial equation for a tetrameric channel, assuming subunit assembly without preference for wild-type or TEA-tagged wild-type subunits (Fig. 5, 

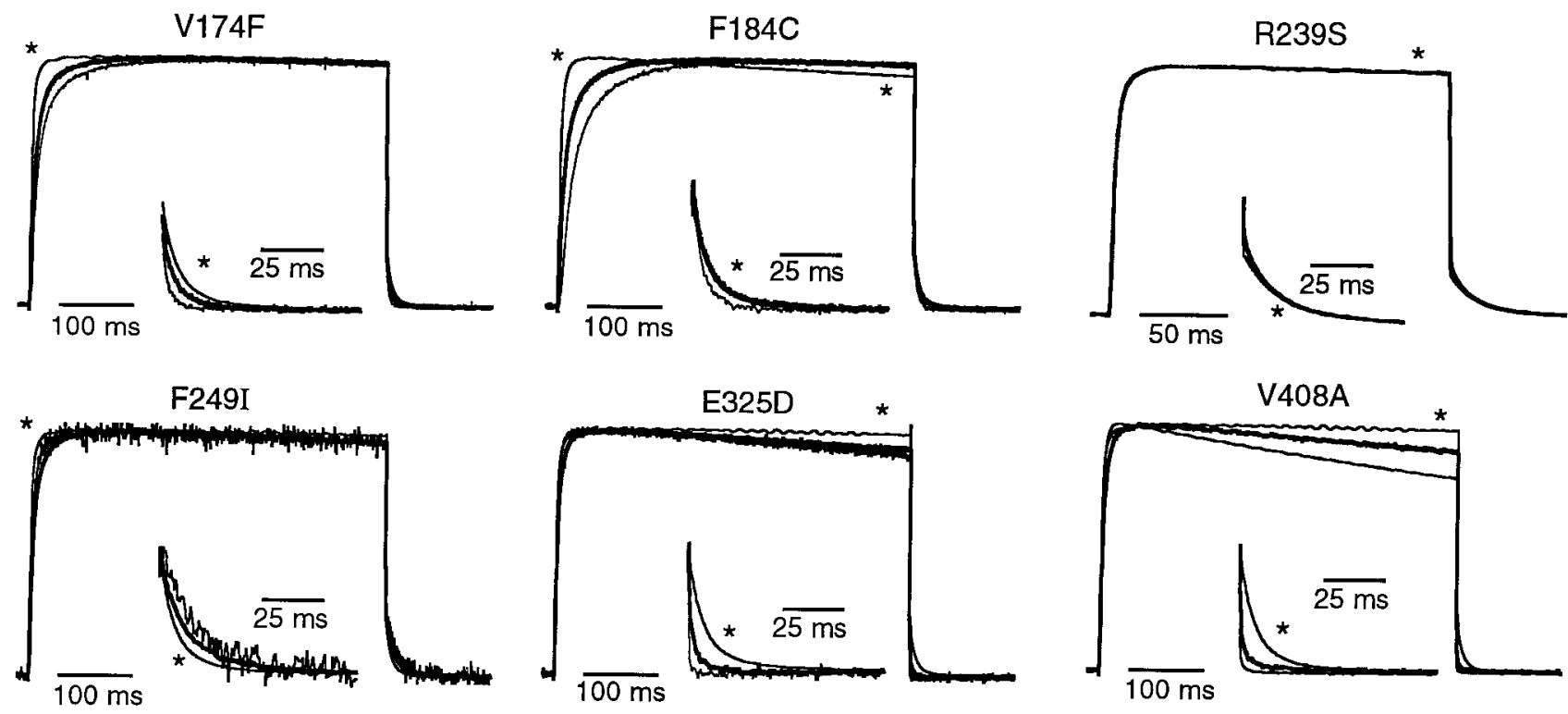

Figure 4. Currents recorded from oocytes coexpressing EA and wild-type subunits. Oocytes were injected with either EA or wild-type mRNA alone (thin traces; * indicates wild type) or coinjected with EA and wild-type mRNA at a 1:1 ratio (thick traces). Currents were evoked at $40 \mathrm{mV}(20 \mathrm{mV}$ for $\mathrm{R} 239 \mathrm{~S}$ ) from a holding potential of $-80 \mathrm{mV}$, and tail currents were recorded at $-50 \mathrm{mV}$. For comparison of kinetics, the traces in each panel were scaled to the same peak. Inset, Enlargement of tail currents recorded at $-50 \mathrm{mV}$.

Table 2. Characterization of currents from oocytes coexpressing wild-type and EA subunits

\begin{tabular}{|c|c|c|c|c|c|}
\hline & \multicolumn{2}{|c|}{ Voltage-dependent parameters } & \multirow{2}{*}{$\begin{array}{l}\text { Activation } \\
\tau_{\text {fast }}(\mathrm{msec})\end{array}$} & \multirow{2}{*}{$\begin{array}{l}\text { Deactivation } \\
\tau(\mathrm{msec})\end{array}$} & \multirow{2}{*}{$\begin{array}{l}\text { C-type } \\
\text { inactivation } \\
I_{\text {final }} / I_{\text {peak }}\end{array}$} \\
\hline & $\mathrm{V}_{1 / 2}(\mathrm{mV})$ & $k(\mathrm{mV})$ & & & \\
\hline Wild type alone & $-28.8 \pm 2.3(6)$ & $8.1 \pm 0.9$ & $2.9 \pm 0.5(5)$ & $11.8 \pm 3.1(21)$ & $0.70 \pm 0.01(10)$ \\
\hline V174F & $-5.3 \pm 3.3(10)^{*}$ & $11.6 \pm 0.9^{*}$ & $5.9 \pm 0.7(4)^{*}$ & $6.2 \pm 0.7(5)^{*}$ & $0.68 \pm 0.02(7)$ \\
\hline F184C & $-13.8 \pm 1.9(8)^{*}$ & $9.5 \pm 0.6^{*}$ & $10.4 \pm 1.1(4)^{*}$ & $11.4 \pm 3.0(8)$ & $0.75 \pm 0.01(4)^{*}$ \\
\hline $\mathrm{R} 239 \mathrm{~S}$ & $-28.0 \pm 2.2(6)$ & $7.8 \pm 1.8$ & $3.7 \pm 0.5(5)$ & $14.1 \pm 1.9(9)$ & $0.72 \pm 0.02(5)$ \\
\hline F249I & $-25.7 \pm 2.1(3)$ & $5.9 \pm 0.8^{*}$ & $4.2 \pm 0.5(3)$ & $14.2 \pm 1.9(8)$ & $0.67 \pm 0.01(3)^{*}$ \\
\hline E325D & $-0.5 \pm 2.6(5)^{*}$ & $17.4 \pm 4.0^{*}$ & $2.5 \pm 0.2(4)$ & $3.3 \pm 0.5(7)^{*}$ & $0.50 \pm 0.04(8)^{*}$ \\
\hline V408A & $-27.5 \pm 2.2(8)$ & $8.2 \pm 0.6$ & $2.6 \pm 0.3(8)$ & $3.7 \pm 1.2(5)^{*}$ & $0.30 \pm 0.01(8)^{*}$ \\
\hline
\end{tabular}

Voltage-dependent parameters of activation $\left(\mathrm{V}_{1 / 2}\right.$ and $\left.k\right)$ were obtained from the Boltzmann equation described in Figure 2 . Time constant for activation at $20 \mathrm{mV}\left(\tau_{\text {fast }}\right)$ and deactivation at $-50 \mathrm{mV}(\tau)$ were determined from exponential fits. C-type inactivation was measured at $20 \mathrm{mV}$ and presented as the ratio $\mathrm{I}_{\text {final }} / \mathrm{I}_{\text {peak }}$. Data are presented as mean $\pm \mathrm{SD}$ (number of cells).

*Significance of $p<0.01$ compared with wild type.

filled circles). The individual $K_{i}$ values used in the binomial equation for heteromeric channels were determined as described previously and are given in Figure 5 (Kavanaugh et al., 1992). The coinjection of equal amounts of wild-type and TEA-tagged V174F, F184C, E325D, or V408A mRNAs yielded TEA dose responses similar to that for wild-type mRNA coinjected with TEA-tagged wild-type mRNA. These results suggest that heteromeric channels are formed from an equal number of wild-type and V174F, F184C, E325D, or V408A subunits. However, the TEA dose response was partially shifted for oocytes coexpressing wild-type and TEAtagged F249I subunits and was not significantly shifted for wild type plus TEA-tagged R239S compared with wild type alone (Fig. 5). Application of the binomial equation to the TEA dose-response curve showed that F249I contributed 0.3 of the total number of subunits available for channel formation. These results are consistent with the possibility that fewer subunits of R239S and F249I are available for coassembly with wild-type subunits.

\section{Reduced amounts of protein for R239S and F249I}

To examine the levels of EA and wild-type subunits in oocytes individually injected with equal amounts of mRNA, total cellular membranes were isolated, and the proteins were prepared as a Western blot and probed with a Kv1.1 polyclonal antibody (Fig. 6). For the representative experiment shown, similar amounts of protein were detected for wild type (1.0), V174F (1.1), F184C (1.0), and V408A (1.0), whereas E325D had slightly reduced levels (0.8). However, oocytes injected with mRNAs for R239S and F249I contained markedly reduced protein levels, 0.4 and 0.2 , respectively, compared with wild type.

\section{DISCUSSION}

EA mutations in Kv1.1 have been examined for functional differences compared with wild-type channels. Four of the functional EA subunits that produce homomeric channels with biophysical properties different from wild-type channels are able to coassemble with wild-type subunits, endowing the resulting heteromeric channels with partial EA phenotypes. These alleles prob- 


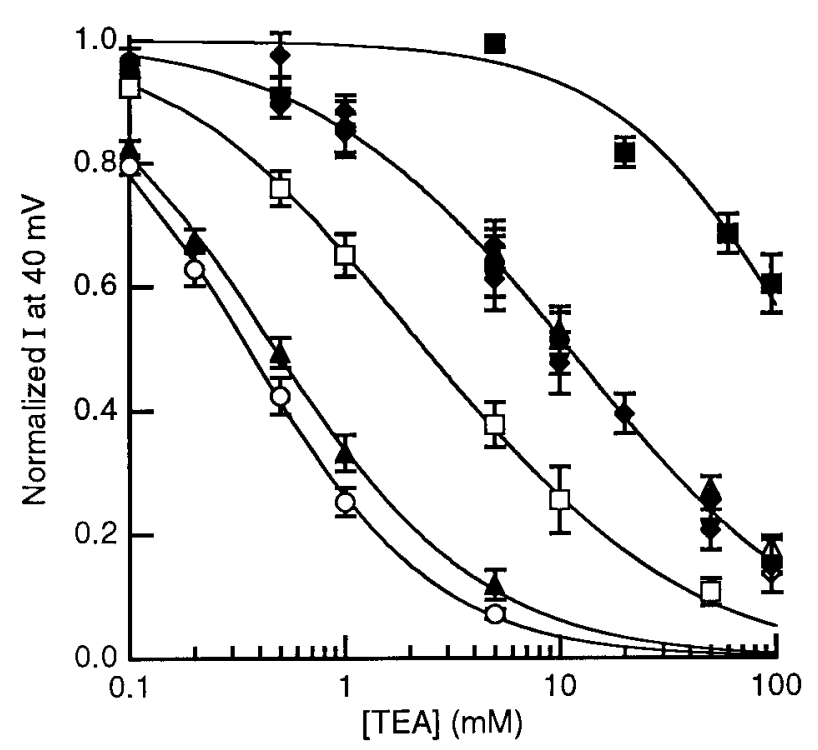

Figure 5. EA subunits coassemble with wild-type subunits. TEA doseresponse curves of oocytes injected with wild-type mRNA alone $(\bigcirc, n=$ 5), TEA-tagged wild-type mRNA alone $(\boldsymbol{\square}, n=7)$, or wild-type mRNA coinjected with either TEA-tagged wild-type $(\bullet, n=7)$ or TEA-tagged EA (R239S, $\boldsymbol{\Delta}, n=4$; F249I, $\square, n=8$; V174F, $\triangle, n=6$; F184C, $\mathbf{\nabla}, n=$ 2; E325D, $\diamond, n=7$; V408A, $\diamond, n=5$ ) mRNAs at a 1:1 ratio. Currents were measured at the end of a $200 \mathrm{msec}$ pulse to $40 \mathrm{mV}$ from a holding potential of $-80 \mathrm{mV}$. Currents in the indicated [TEA] were normalized by the control current. Data points for wild type were fitted according to the equation: $I=K_{i} /\left([\mathrm{TEA}]+K_{i}\right)$, giving a $K_{i}$ for TEA of $0.4 \mathrm{~mm}$. Data points from the coinjection of wild type with TEA-tagged wild type, TEA-tagged R239S, or TEA-tagged F249I were fitted according to the binomial equation:

$$
I=\sum_{n=0}^{4} C_{n}^{4} \cdot f^{4-n} \cdot(1-f)^{n} \cdot K_{i, n} /\left([\mathrm{TEA}]+K_{i, n}\right)
$$

where $f$ equals the fraction of wild-type subunits, $C_{n}^{4}=4 ! / n !(4-n) !$, and the individual $K_{i, n}$ values were $0.4,1.7,9.8,25.3$, and $123.8 \mathrm{~mm}$ for $n=$ $0-4$, respectively. The values of $f$ for wild type coexpressed with TEAtagged subunits of R239S, F249I, and wild type were 0.94, 0.70, and 0.45, respectively. The TEA dose response of homomeric TEA-tagged EA subunits was similar to that of homomeric TEA-tagged wild-type subunits; the EA mutations do not alter TEA sensitivity. Error bars indicate SD.

ably affect the symptoms of the disorder through dominant negative interactions with wild-type subunits as well as the intrinsic differences between homomeric EA and wild-type channels. F249I, which also coassembles with wild-type subunits, shows reduced levels of protein and may affect EA symptoms by both dominant negative and haplotype insufficiency mechanisms. Finally, R239S produces reduced but significant levels of protein but does not form functional homomeric channels and does not coassemble with wild-type subunits, suggesting that the R239S allele results in EA because of too few Kv1.1 subunits, haplotype insufficiency.

Several EA subunits yielded homomeric channels with gating properties distinct from wild-type channels. Interestingly, EA mutations in the same domain of the subunit gave similar phenotypes. V174F and F184C reside in the first transmembrane domain, and both showed a shift in voltage dependence of activation of $\sim 30 \mathrm{mV}$; F184C also had slower activation kinetics. E325D and V408A, residing on opposite sides of the deep pore in S5 and S6, respectively, both showed accelerated activation and deactivation

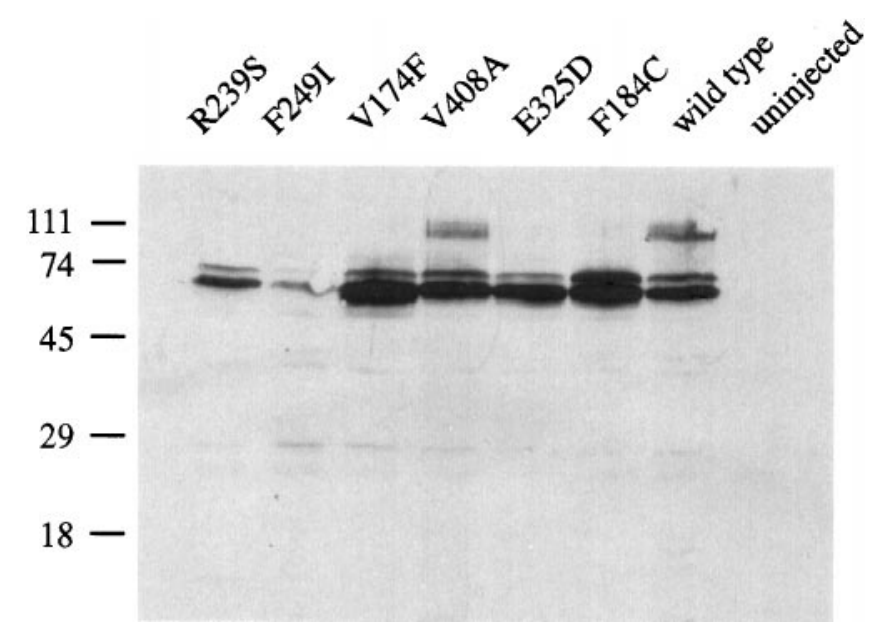

Figure 6. Western blot of membranes from oocytes expressing EA or wild-type subunits. Oocytes were injected with equal amounts of wildtype or EA mRNAs. Total membranes were prepared from equal numbers of oocytes for each sample, and a protein assay based on a Bradford procedure was used to quantify the protein concentration. Equal amounts of protein, representing approximately five oocytes, were loaded on each lane and probed with the Kv1.1 polyclonal antibody. The ladder on the left is in kilodaltons. Doublets were observed at $\sim 60 \mathrm{kDa}$, near the calculated molecular weight for Kv1.1, which probably reflects glycosylated and unglycosylated forms. The additional band detected at $\sim 110 \mathrm{kDa}$ for V408A and wild type may be a result of to subunit dimerization. Among the six EA mutants studied, R239S, F249I, and E325D showed lower amounts of protein compared with wild type.

kinetics and faster C-type inactivation. Additionally, for E325D the voltage dependence was shifted by $\sim 60 \mathrm{mV}$, and both activation and deactivation kinetics were less steeply voltagedependent. The absence or marked reduction of currents for R239S and F249I, respectively, together with reduced amounts of protein, suggest that the intracellular loop between transmembrane domains 2 and 3 is critical for channel biosynthesis and subunit stability.

To examine whether EA subunits coassembled with wild-type subunits, mRNAs were coinjected in a 1:1 ratio, mimicking the in vivo heterozygous condition. Current amplitudes from coinjected oocytes were reduced compared with oocytes injected with the same total amount of wild-type mRNA. In general, the altered functions seen from homomeric EA channels were also observed in coinjected oocytes, although the magnitude of the effects was less than in homomeric EA channels, suggesting that heteromeric channels were formed. Indeed, current measurements from oocytes coexpressing wild-type and EA subunits made less sensitive to TEA (TEA-tagged) clearly demonstrated that the EA mutants V174F, F184C, E325D, and V408A coassemble with wild type. The TEA dose-response curves for these TEA-tagged EA subunits coexpressed with wild type were similar to that seen for coexpression of wild-type and TEA-tagged wild-type subunits. Accordingly, this suggests that equal numbers of these EA and wild-type subunits are available for channel assembly. However, the diminished current amplitudes of homomeric V174F, F184C, and E325D channels, which are partially rescued when these subunits are coexpressed with wild-type subunits, are consistent with either a reduction in single-channel current and/or the total number of channels. A reduction in the single-channel current caused by EA subunits may be conferred on heteromeric channels with different potency depending on the stoichiometry of EA and wild-type subunits. This would have been realized as a reduced 
shift in the TEA dose-response experiments, which was not seen for V174F, F184C, E325D, or V408A. In contrast, protein levels for V174F, F184C, and V408A subunits, and to a lesser extent E325D subunits, were similar to those of wild type, suggesting that heteromeric channels may not move to the cell surface as readily as wild-type channels. Therefore, these EA mutations may affect the symptoms of the disorder through both dominant negative (altered channel function) and haplotype insufficiency effects (reduced currents).

R239S and F249I may affect channel function in different ways. Expression of TEA-tagged F249I subunits shifted the TEA sensitivity approximately two-thirds of that seen for coexpression for wild-type and TEA-tagged wild-type subunits, although R239S subunits had no effect. Western blot analyses, using total cellular membranes, showed reduced levels of protein compared with wild type for R239S (0.4) and F249I (0.2). There is more protein for R239S than for F249I, yet R239S has no obvious effects either when expressed alone (no current) or together with wild type (no altered functions and no shift in TEA sensitivity). In contrast, F249I channels are functional and only modestly distinguished from wild-type channels, and coexpression with wild-type subunits shifts TEA sensitivity. These results suggest that F249I subunits are relatively unstable, but intact subunits coassemble with wild-type subunits. Therefore, F249I subunits may demonstrate reduced current amplitudes when expressed alone or with wild-type subunits because of a reduction in the number of channels in the membrane as well as possible effects on the single-channel currents. In contrast, R239S subunits do not appear to form functional channels when expressed alone and do not seem to coassemble with wild-type subunits, even though there is more total cellular protein than seen for F249I. These results suggest that R239S subunits are trapped inside the cell, unavailable for coassembly with wild-type subunits, and that the R239S mutation gives rise to EA symptoms through haplotype insufficiency.

The EA E325D mutation is remarkable in several ways. This position, which affects gating, is in a domain implicated as part the internal pore region of Shaker-like potassium channels (Slesinger et al., 1993), suggesting that E325 may participate in both gating and conduction processes. The E325D mutation resides at the intracellular border of S5 and differs from wild type by a single side chain carbonyl. Yet E325D induces a $60 \mathrm{mV}$ shift in activation voltage and a reduction in slope; more energy is required for channel activation compared with wild-type channels. It is possible that a $\mathrm{D}$ in this position enables the side chain to form a salt bridge otherwise not available when E occupies this position, and the strong gating effects suggest that the counter charge may be one of the positively charged residues in S4. The E325D phenotype is strikingly similar to those seen after neutralization of E283 or D316 in Shaker. For D316, there is evidence that this residue interacts with at least one of the positively charged residues in S4 (Papazian et al., 1995; Seoh et al., 1996). These and other results suggest that residues in S4 form salt bridges with residues in S2 and S3 (Papazian and Bezanilla, 1997). However, the E325D phenotype implicates additional domains beyond S2 and S3 that may couple with S4.
These studies indicate that, depending on the location within the Kv1.1 subunit, different parameters of channel function are affected, and that each EA allele may result in different symptomatic severity. Clinically, however, EA patients within a family and among families do not show a pattern of severity that correlates with the underlying alleles. This is likely caused by the heterogeneous genetic background in humans. In contrast, introduction of different EA alleles into an isogenic mouse background may reveal symptomatic differences obviated by the heterologous expression studies presented here.

\section{REFERENCES}

Ackerman MJ, Clapham DE (1997) Mechanisms of disease. N Engl J Med 336:1575-1586.

Adelman JP, Bond CT, Pessia M, Maylie J (1995) Episodic ataxia results from voltage-dependent potassium channels with altered functions. Neuron 15:1449-1454.

Ashizawa T, Butler IJ, Harati Y, Roongta SM (1983) A dominantly inherited syndrome with continuous motor neuron discharges. Ann Neurol 13:285-290.

Browne DL, Gancher ST, Nutt JG, Brunt ERP, Smith EA, Kramer P, Litt M (1994) Episodic ataxia/myokymia syndrome is associated with point mutations in the human potassium channel gene, KCNA1. Nat Genet 8:136-140.

Browne DL, Brunt ERP, Griggs RC, Nutt JG, Gancher ST, Smith EA, Litt M (1995) Identification of two new KCNA1 mutations in episodic ataxia/myokymia families. Hum Mol Genet 4:1671-1672.

Brunt ER, Weeden TWV (1990) Familial paroxysmal kinesigenic ataxia and continuous myokymia. Brain 113:1361-1382.

Gancher S, Nutt J (1986) Autosomal dominant episodic ataxia: a heterogeneous syndrome. Mov Disord 1:239-253.

Geering K, Theulaz I, Verrey F, Hauptle MT, Rossier BC (1989) A role for the beta-subunit in the expression of functional $\mathrm{Na}^{+}-\mathrm{K}^{+}$ATPase in Xenopus oocytes. Am J Physiol 257:851-858.

Greenberg DA (1997) Calcium channels in neurological disease. Ann Neurol 42:275-282.

Kavanaugh MP, Hurst RS, Yakel J, Varnum MD, Adelman JP, North RA (1992) Multiple subunits of a voltage-dependent potassium channel contribute to the binding site for tetraethylammonium. Neuron 8:1-20.

Kraus JE, McNamara JO (1995) Clinical relevance of defects in signalling pathways. Curr Opin Neurobiol 5:358-366.

Litt M, Kramer P, Browne D, Gancher S, Brunt ER, Root D, Phromchotikul T, Dubay CJ, Nutt J (1994) A gene for episodic ataxia/myokymia maps to chromosome 12p13. Am J Hum Genet 55:702-709.

MacKinnon R (1991) Determination of the subunit stoichiometry of a voltage-activated potassium channel. Nature 350:232-235.

MacKinnon R, Yellen G (1990) Mutations affecting TEA blockade and ion permeation in voltage-activated $\mathrm{K}^{+}$channels. Science 250:276-279.

Papazian DM, Bezanilla F (1997) How does an ion channel sense voltage? News Physiol Sci 12:203-210.

Papazian DM, Shao XM, Seoh SA, Mock AF, Huang Y, Wainstock DH (1995) Electrostatic interactions of S4 voltage sensor in Shaker $\mathrm{K}^{+}$ channel. Neuron 14:1293-1301.

Seoh SA, Sigg D, Papazian DM, Bezanilla F (1996) Voltage-sensing residues in the S2 and $\mathrm{S} 4$ segments of the Shaker $\mathrm{K}^{+}$channel. Neuron 16:1159-1167.

Slesinger PA, Jan YN, Jan LY (1993) The S4-S5 loop contributes to the ion-selective pore of potassium channels. Neuron 11:739-749.

Tucker SJ, Bond CT, Herson P, Pessia M, Adelman JP (1996) Inhibitory interactions between two inward rectifier $\mathrm{K}^{+}$subunits mediated by the transmembrane domains. J Biol Chem 271:5866-5870. 